\title{
INTEGRABILITY ALONG A LINE FOR A CLASS OF ENTIRE FUNCTIONS
}

BY

\author{
R. P. BOAS, JR.(')
}

1. Introduction. Duffin and Schaeffer have recently shown [5] that if $f(z)$ is an entire function of exponential type $c<\pi$ such that

$$
\sum_{-\infty}^{\infty}\left|f\left(\lambda_{n}\right)\right|^{2}<\infty,
$$

then for some $K$ (independent of $f(z)$ )

$$
\int_{-\infty}^{\infty}|f(x)|^{2} d x \leqq K \sum_{-\infty}^{\infty}\left|f\left(\lambda_{n}\right)\right|^{2}
$$

provided that $\left\{\lambda_{n}\right\}$ is an increasing sequence of real numbers such that $\left|\lambda_{n}-n\right|<L$ and $\left|\lambda_{n+1}-\lambda_{n}\right| \geqq \delta>0$. This is the $L^{2}$-analogue of the theorem, proved in different ways by Duffin and Schaeffer [6] and by B. Levin [8], that $f(x)$ is bounded if $\left\{f\left(\lambda_{n}\right)\right\}$ is bounded; the cases of the two theorems when $\lambda_{n}=n$ are due respectively to Plancherel and Pólya [11] and Cartwright [3].

The object of this paper is to replace (1.1), (1.2) respectively by

$$
\begin{gathered}
\sum_{-\infty}^{\infty} \phi\left(\left|f\left(\lambda_{n}\right)\right|\right)<\infty \\
\int_{-\infty}^{\infty} \phi(H|f(x)|) d x \leqq K \sum_{-\infty}^{\infty} \phi\left(\left|f\left(\lambda_{n}\right)\right|\right),
\end{gathered}
$$

with constants $H$ and $K$ independent of $f(z)$, for a wide class of functions $\phi(t)$ which includes $t^{p}$ for $p>0$. The proof is quite different from that given by Duffin and Schaeffer for $\phi(t)=t^{2}$; it involves a combination of ideas used by Plancherel and Pólya for $\lambda_{n}=n, \phi(t)=t^{p}$; an interpolation formula of Valiron; and Levin's estimates for a product with zeros at the $\lambda_{n}$.

The precise statement of the theorem is as follows.

THEOREM. Let $f(z)$ be an entire function of exponential type such that

$$
\lim \sup y^{-1} \log |f(i y) f(-i y)|=2 c<2 \pi .
$$

Let $\left\{\lambda_{n}\right\}$ be a sequence of complex numbers, with $\lambda_{0}=0,\left|\lambda_{n}-n\right| \leqq L,\left|\lambda_{n+m}-\lambda_{n}\right|$ $>2 \delta>0(m \neq 0)$. Let $\beta(x)$ be a nondecreasing function such that $\mid \beta(x+L)$

Presented to the Society, February 23, 1952; received by the editors December 20, 1951.

(1) The Author is a Fellow of the John Simon Guggenheim Memorial Foundation. 
$-\beta(x-L) \mid \leqq b$ and (if $L<1)|\beta(x+1 / 2)-\beta(x-1 / 2)| \leqq b$. Let $\phi(x)$ be either (i) an increasing convex nonnegative function or (ii) an increasing nonnegative continuous function such that $\phi(0)=0, \phi(\infty)=\infty, \phi(x) / x$ decreases, and

$$
\phi(x y) \leqq \phi(x) \phi(y) \text {. }
$$

Then if $\sum_{-\infty}^{\infty} \phi\left(\left|f\left(\lambda_{n}\right)\right|\right)$ converges, there are numbers $H$ and $K$, depending only on $c, L, \delta, b$, and $\phi$, such that

$$
\int_{-\infty}^{\infty} \phi(H|f(x)|) d \beta(x) \leqq K \sum_{-\infty}^{\infty} \phi\left(\left|f\left(\lambda_{n}\right)\right|\right) ;
$$

in case (i), $K \leqq b$; in case (ii), $H \geqq 1$.

If $\phi(x)=x^{p}$, we have case (i) if $p \geqq 1$, case (ii) if $0<p<1$. In case (i) we can also, for example, take $\phi(x)=e^{-1 / x}$ for $x$ near 0 and it follows that if $\sum \exp \left\{-1 /\left|f\left(\lambda_{n}\right)\right|\right\}$ converges, then $\int_{-\infty}^{\infty} \exp \{-H /|f(x)|\} d x$ converges for some $H$, a result which is new even when $\lambda_{n}=n$. Functions satisfying (1.6) have been discussed by Cooper [4], who showed (in particular) that they satisfy $\phi(x) \leqq A x^{\alpha}$ for $x$ near 0 .

A converse theorem, when $\phi(t)=t^{p}$, was given by Plancherel and Pólya [11]: the convergence of

$$
\int_{-\infty}^{\infty} \phi\{|f(x)|\} d x
$$

implies that of

$$
\sum_{n=-\infty}^{\infty} \phi\left\{e^{-c \delta}\left|f\left(\lambda_{n}\right)\right|\right\}
$$

assuming only that the $\lambda_{n}$ are real and $\left|\lambda_{n+m}-\lambda_{n}\right|>2 \delta>0, m \neq 0$, with no restriction on the type of $f(z)$ (except that it is finite). It is possible to modify the direct theorem, by using a Stieltjes integral instead of a sum on the right of (1.7), so that it includes this converse theorem. However, the converse actually holds for a wider class of functions $\phi$ than that considered in the theorem, namely when $\phi(t)$ is an increasing non-negative convex function of $\log t$. A proof can be given by a modification of Plancherel and Pólya's method, but I shall not give it here. I note only the lemma that

$$
\int_{-\infty}^{\infty} \phi\left\{e^{-c|y|}|f(x+i y)|\right\} d x \leqq \int_{-\infty}^{\infty} \phi\{|f(x)|\} d x,
$$

and the form of the final result for real $\lambda_{n}$ :

$$
\sum_{n=-\infty}^{\infty} \phi\left\{e^{-c \delta}\left|f\left(\lambda_{n}\right)\right|\right\} \leqq K \int_{-\infty}^{\infty} \phi\{|f(x)|\} d x,
$$


where $f(z)$ is of type $c$ and $K$ depends only on $\delta$.

2. An interpolation formula. We need the following lemmas.

LEMMA 1. Let

$$
G(z)=z \prod_{n=1}^{\infty}\left(1-z / \lambda_{n}\right)\left(1-z / \lambda_{-n}\right),
$$

where $\left\{\lambda_{n}\right\}$ satisfies the conditions of the theorem. Then $G(z)$ is an entire function of exponential type $\pi$, satisfying

$$
\begin{aligned}
|G(x+i y)| & \leqq A(|x|+1)^{4 L}, & |y| \leqq 3 L ; \\
\left|G^{\prime}\left(\lambda_{k}\right)\right| & \geqq C\left(1+\left|\lambda_{k}\right|\right)^{-4 L-1}, &
\end{aligned}
$$

where $A$ and $C(>0)$ depend only on $\delta$ and $L$.

Lemma 1 was proved by Levin [8]; less sharp estimates which would, however, be entirely adequate for our present purposes (and also for proving the boundedness of $f(x)$ when $\left\{f\left(\lambda_{n}\right)\right\}$ is bounded) were given much earlier by Levinson [10].

LemMa 2. For $\left|z-\lambda_{n}\right|>\delta / 2$, and each positive $\sigma$,

$$
\exp \{\pi r(|\sin \theta|-\sigma)\} /\left|G\left(r e^{i \theta}\right)\right|=O(1) .
$$

Lemma 2, which is true whenever $\left\{\lambda_{n}\right\}$ has density 1 and satisfies the separation condition of the theorem, follows from a theorem proved by Levinson [9, pp. 92-93].

We may suppose instead of (1.5) that lim sup $|y|^{-1} \log |f(i y)|=c<\pi$ as $y \rightarrow \pm \infty$, since otherwise we can consider $f(z) e^{i \alpha z}$ with a suitable real $\alpha$; and then it follows by a Phragmén-Lindelöf argument that

$$
\left|f\left(r e^{i \theta}\right)\right|=O\left(e^{r\left(c^{\prime}|\sin \theta|+d|\cos \theta|\right)}\right), \quad r \rightarrow \infty,
$$

for some $c^{\prime}<\pi$ and finite $d$, and each $\theta$.

Now let $q$ be an integer, not less than $4 L+3$, and put

$$
H(z)=\sum_{k=-\infty}^{\infty} \frac{G(z) f\left(\lambda_{k}\right)}{G^{\prime}\left(\lambda_{k}\right)\left(z-\lambda_{k}\right)}\left(\frac{z}{\lambda_{k}}\right)^{q},
$$

where the prime indicates the omission of the term with $k=0$. Let $P(z)$ be the sum of the first $q+1$ terms of the Maclaurin series of $z f(z) / G(z)$, and put

$$
\psi(z)=\frac{f(z)-H(z)-G(z) P(z) / z}{z^{q} G(z)} .
$$

In the first place, since $\phi(x) \uparrow \infty$ when $x \uparrow \infty$, the convergence of $\sum \phi\left(\left|f\left(\lambda_{n}\right)\right|\right)$ implies that the numbers $f\left(\lambda_{n}\right)$ are bounded; hence by (2.2) and our choice of $q$, the series for $H(z)$ converges uniformly in any bounded region; hence $H(z)$ 
is an entire function taking the values $f\left(\lambda_{k}\right)$ at $z=\lambda_{k}, k \neq 0$. Since $f(z)$ $-G(z) P(z) / z$ has a $(q+1)$-fold zero at $0, \psi(z)$ is also entire, and it is easy to verify that it is actually of exponential type.

We next estimate $\psi\left(r e^{i \theta}\right)$ more precisely for large $r$ and $\theta$ near $\pm \pi / 2$. We have

$$
|\psi(z)| \leqq\left|\frac{f(z)}{z^{q} G(z)}\right|+\left|\frac{H(z)}{z^{q} G(z)}\right|+\left|\frac{P(z)}{z^{q+1}}\right|=T_{1}+T_{2}+T_{3} .
$$

Then $T_{3}=o(1)$ since $P(z)$ is of degree at most $q$. For $T_{1}$ we have by (2.3) and (2.4)

$$
\begin{aligned}
T_{1} & \leqq M e^{r\left(c^{\prime}|\sin \theta|+d|\cos \theta|\right)-\pi r(|\sin \theta|-\sigma)} \\
& =M e^{r(-\beta|\sin \theta|+d|\cos \theta|+\sigma)},
\end{aligned}
$$

where $\beta>0$, and $M$ depends on $f(z), \sigma$, and $\theta$, while $\sigma$ is arbitrarily small; thus $T_{1}$ is bounded on $\arg z=\theta$ if $\theta$ is so near $\pm \pi / 2$ that $-\beta|\sin \theta|+d|\cos \theta|$ $+\sigma<0$. For $T_{2}$ we have by $(2.2)$

$$
T_{2} \leqq R \sum_{k=-\infty}^{\infty} \frac{\left(1+\left|\lambda_{k}\right|\right)^{4 L+1}}{\left|z-\lambda_{k}\right|\left|\lambda_{k}\right|^{q}}
$$

where $R$ is a constant, and this is bounded for $|y| \geqq 2 L$, say, since $\left|\Im\left(\lambda_{k}\right)\right| \leqq L$ and $q \geqq 4 L+3$.

Hence $\psi(z)$ is an entire function of exponential type bounded on four rays, any two consecutive ones of which make an angle of less than $\pi$, and hence by a Phragmén-Lindelöf theorem $\psi(z)$ is bounded everywhere and so is a constant. Finally, this constant must be zero, since for $z=i y,|y| \rightarrow \infty$, $T_{1} \rightarrow 0, T_{3} \rightarrow 0$, and $T_{2} \rightarrow 0$ because $\left|i y-\lambda_{k}\right| \rightarrow \infty$ uniformly in (2.5).

Thus we have the formula

$$
f(z)=\sum_{k=-\infty}^{\infty} \frac{G(z) f\left(\lambda_{k}\right)}{G^{\prime}\left(\lambda_{k}\right)\left(z-\lambda_{k}\right)}\left(\frac{z}{\lambda_{k}}\right)^{q}+z^{-1} G(z) P(z),
$$

which is due to Valiron [12]. (Valiron's hypotheses are somewhat different, and it is somewhat easier to follow the lines of Valiron's proof with our hypotheses than to verify that our hypotheses imply his.) The same formula was established by Ahiezer [1] by another method under more general hypotheses which are irrelevant for our present purposes; the same degree of generality can be attained by Valiron's method by using a lemma of Boas [2, p. 479].

3. Proof of the theorem. We shall now use for the first time the fact that the constants $A$ and $C$ of Lemma 1 depend only on $L$ and $\delta$ and are otherwise independent of the position of the $\lambda_{n}$.

Let $m$ be an integer and put $\lambda_{n}^{(m)}=\lambda_{n+m}-\lambda_{m}$, so that $\lambda_{0}^{(m)}=0,\left|\lambda_{n}^{(m)}-n\right|$ $\leqq 2 L$, and $\left|\lambda_{n}^{(m)}-\lambda_{k}^{(m)}\right| \geqq 2 \delta$. Let $G_{m}(z)$ denote the function of Lemma 1 , 
formed with $\lambda_{n}^{(m)}$ instead of $\lambda_{n}$. Then $G_{m}(z)$ satisfies (2.1) and (2.2), with $L$ replaced by $2 L, A$ and $C$ being independent of $m$. Let $h_{m}(z)=f\left(z+\lambda_{m}\right) \sin ^{q} \eta z$, where now $q \geqq 8 L+3$ in case (i), and in case (ii) $q$ is so large that $\sum \phi\left(k^{8 L+2-q}\right)$ converges; the latter choice is possible because $\phi(x) \leqq A x^{\alpha}$ for small $x$ and some positive $\alpha$. If $\eta$ is so small that $q \eta<\pi-c, h_{m}(z)$ is of type less than $\pi$; and $\left|h_{m}\left(\lambda_{n}^{(m)}\right)\right| \leqq\left|f\left(\lambda_{n+m}\right)\right|=O(1)$. Hence (2.6) applies to $h_{m}(z)$. Since $P(z)$ is of degree $q$ at most, and $h_{m}(z)$ has a $q$-fold zero at $0, P(z) \equiv 0$ in this case and we have

$$
h_{m}(z)=\sum_{k=-\infty}^{\infty} \frac{G_{m}(z) h_{m}\left(\lambda_{k}^{(m)}\right)}{G_{m}^{\prime}\left(\lambda_{k}^{(m)}\right)\left(z-\lambda_{k}^{(m)}\right)}\left(\frac{z}{\lambda_{k}^{(m)}}\right)^{q} .
$$

We may suppose that $L \geqq 1$ and $\eta<1 / L$. By the maximum principle, if $\Gamma$ is the boundary of the square of side $4 L$ with center at the origin and sides parallel to the coordinate axes, and $\mu_{m}$ is the maximum of $\left|f\left(z+\lambda_{m}\right)\right|$ for $z$ inside $\Gamma, \mu_{m} \leqq \max _{\Gamma}\left|f\left(z+\lambda_{m}\right)\right|$. On $\Gamma,\left|\sin ^{q} z\right|$ has a positive minimum, $1 / \gamma$; and $\left|h\left(\lambda_{k}^{(m)}\right)\right| \leqq\left|f\left(\lambda_{k+m}\right)\right| ;$ so

$$
\mu_{m} \leqq \gamma C^{-1} \sum_{k=-\infty}^{\infty} \max _{\Gamma}\left|\frac{G_{m}(z)}{z-\lambda_{k}^{(m)}}\right| \frac{\left|f\left(\lambda_{k+m}\right)\right|\left(1+\left|\lambda_{k}^{(m)}\right|\right)^{8}(3 L)^{q}}{\left|\lambda_{k}^{(m)}\right|^{q}} .
$$

If $z \in \Gamma$ and $\left|z-\lambda_{k}^{(m)}\right| \geqq 1$, by (2.1) we have

$$
\max _{\Gamma}\left|\frac{G_{m}(z)}{z-\lambda_{k}^{(m)}}\right| \leqq A(3 L)^{8 L} .
$$

On the other hand, if $z \in \Gamma$ and $\left|z-\lambda_{k}^{(m)}\right|<1$, then $G_{m}(z) /\left(z-\lambda_{k}^{(m)}\right)$ is regular on $\Gamma$, and the maximum of its absolute value does not exceed

$$
\max _{z \in \Gamma} \max _{|z-w|=1}\left|\frac{G_{m}(z)}{z-w}\right| \leqq A(3 L+1)^{8 L}
$$

by (2.1). Hence we have

$$
\mu_{m} \leqq N \sum_{k=-\infty}^{\infty}\left|f\left(\lambda_{k+m}\right)\right| b_{k}^{(m)},
$$

where $b_{k}^{(m)}=\left(1+\left|\lambda_{k}^{(m)}\right|\right)^{8 L+1}\left|\lambda_{k}^{(m)}\right|^{-q}$ and $N$ depends only on $L$ and $\delta$ (not on $m$ ). Now for $k \neq 0,\left|\lambda_{k}^{(m)}\right| \geqq \delta$ and $k+2 L \geqq\left|\lambda_{k}^{(m)}\right| \geqq k-2 L$; hence

$$
\begin{array}{ll}
b_{k}^{(m)} \leqq(1+k+2 L)^{8 L+1}(k-2 L)^{-q}, & |k|>2 L ; \\
b_{k}^{(m)} \leqq(1+4 L)^{8 L+1} \delta^{-q}, & |k| \leqq 2 L ;
\end{array}
$$

and so, by our choice of $q$, we have $b_{k}^{(m)} \leqq b_{k}$, where $b_{k}$ is independent of $m$ and $\sum b_{k}$ converges in case (i), $\sum \phi\left(b_{k}\right)$ converges in case (ii). 
Thus from (3.3) we have (with $b_{0}=0$ )

$$
\mu_{m} \leqq N \sum_{k=-\infty}^{\infty}\left|f\left(\lambda_{k+m}\right)\right| b_{k}=N \sum_{n=-\infty}^{\infty}\left|f\left(\lambda_{n}\right)\right| b_{n-m} .
$$

From this the theorem follows easily. Let $1 / H=N \sum_{-\infty}^{\infty} b_{k}$. Then in case (i) we have by the properties of convex functions $[7, \mathrm{p} .72]$

$$
\phi\left(H \mu_{m}\right)=\phi\left(H \sum_{n=-\infty}^{\infty}\left|f\left(\lambda_{n}\right)\right| N b_{n-m}\right) \leqq H \sum_{n=-\infty}^{\infty} N b_{n-m} \phi\left(\left|f\left(\lambda_{n}\right)\right|\right) ;
$$

hence

$$
\sum_{m=-\infty}^{\infty} \phi\left(H \mu_{m}\right) \leqq \sum_{n=-\infty}^{\infty} \phi\left(\left|f\left(\lambda_{n}\right)\right|\right)
$$

and

$$
\begin{aligned}
\int_{-\infty}^{\infty} \phi(H|f(x)|) d \beta(x) & \leqq \sum_{m=-\infty}^{\infty} \int_{-L}^{L} \phi(H|f(x+m)|) d \beta(x) \\
& \leqq b \sum_{m=-\infty}^{\infty} \phi\left(H \max _{-L \leqq x \leqq L}|f(x+m)|\right) \\
& =b \sum_{m=-\infty}^{\infty} \phi\left(H \max _{-L \leqq x \leqq L}\left|f\left(x+\lambda_{m}+m-\lambda_{m}\right)\right|\right) \\
& \leqq b \sum_{m=-\infty}^{\infty} \phi\left(H \mu_{m}\right) \leqq b \sum_{n=-\infty}^{\infty} \phi\left(\left|f\left(\lambda_{n}\right)\right|\right),
\end{aligned}
$$

which is the conclusion of the theorem in case (i).

In case (ii) we return to (3.4) and now we have, first since $\phi(x) / x$ decreases $[7$, p. 83] and then by (1.6),

$$
\begin{aligned}
\phi\left(\mu_{m}\right) \leqq & \sum_{n=-\infty}^{\infty} \phi\left(N\left|f\left(\lambda_{n}\right)\right| b_{n-m}\right) \leqq \sum_{n=-\infty}^{\infty} \phi\left(\left|f\left(\lambda_{n}\right)\right|\right) \phi\left(b_{n-m}\right) \phi(N), \\
& \sum_{m=-\infty}^{\infty} \phi\left(\mu_{m}\right) \leqq \phi(N) \sum_{n=-\infty}^{\infty} \phi\left(\left|f\left(\lambda_{n}\right)\right|\right) \sum_{m=-\infty}^{\infty} \phi\left(b_{m}\right),
\end{aligned}
$$

and the conclusion follows as before by starting from this inequality instead of (3.5).

\section{REFERENCES}

1. N. I. Ahiezer, Ob interpolirovanii tselyh trantsendentnyh funktsit konechnot stepeni (On the interpolation of entire transcendental functions of finite degree), Doklady Akad. Nauk SSSR. N.S. vol. 65 (1949) pp. 781-784. 481 .

2. R. P. Boas, Jr., The growth of analytic functions, Duke Math. J. vol. 13 (1946) pp. 471- 
3. M. L. Cartwright, On certain integral functions of order one, Quart. J. Math. Oxford Ser. vol. 7 (1936) pp. 46-55.

4. R. Cooper, The converse of the Cauchy-Hölder inequality and the solutions of the inequality $g(x+y) \leqq g(x)+g(y)$, Proc. London Math. Soc. (2) vol. 26 (1927) pp. 415-432.

5. R. J. Duffin and A. C. Schaeffer, $A$ class of nonharmonic Fourier series, Trans. Amer. Math. Soc. vol. 72 (1952) pp. 341-366.

6. - , Power series with bounded coefficients, Amer. J. Math. vol. 67 (1945) pp. 141154.

7. G. H. Hardy, J. E. Littlewood, and G. P6lya, Inequalities, Cambridge, 1934.

8. B. Levin, O funktsiyah konechnot stepeni, ogranichennyh na posledovatelnosti tochek (On functions of finite degree, bounded on a sequence of points), Doklady Akad. Nauk SSSR. N.S. vol. 65 (1949) pp. 265-268.

9. N. Levinson, Gap and density theorems, Amer. Math. Soc. Colloquium Publications, vol. 26. New York, 1940.

10. —, On a problem of Polya, Amer. J. Math. vol. 58 (1936) pp. 791-798.

11. M. Plancherel and G. P6lya, Fonctions entières et intégrales de Fourier multiples, Comment. Math. Helv. vol. 9 (1937) pp. 224-248; vol. 10 (1938) pp. 110-163.

12. G. Valiron, Sur la formule d'interpolation de Lagrange, Bull. Sci. Math. (2) vol. 49 (1925) pp. 181-192, 203-224.

NORTHWESTERN UNIVERSITY, Evanston, ILL. 